\section{OP0136 SIBLINGS OF PATIENTS WITH RHEUMATOID ARTHRITIS ARE AT INCREASED RISK OF ACUTE CORONARY SYNDROME}

H. Westerlind ${ }^{1}$, M. Holmqvist ${ }^{1}$, L. Ljung ${ }^{1,2}$, T. Frisell ${ }^{1}$, J. Askling ${ }^{1} .{ }^{1}$ Department of Medicine, Clinical Epidemiology Unit, Karolinska Institutet, Stockholm; ${ }^{2}$ Department of Public Health and Clinical Medicine/Rheumatology, Umeå University, Umeå, Sweden

Background: Patients with rheumatoid arthritis (RA) are at increased risk of cardiovascular disease such as acute coronary syndromes (ACS), which cannot entirely be explained by traditional cardiovascular risk factors. Studies have shown an association between RA disease severity and risk of ACS, speaking for a contribution of the RA disease per se to the excess ACS risk. In a recent report, however, we demonstrated that despite more efficient control of inflammation in RA during the recent years, the excess risk for ACS among patients with RA compared to the general population remains ${ }^{1}$. This finding suggests that besides effects related to the RA disease per se, there may be a shared susceptibility. If the excess risk of ACS in patients with RA were increased due to this, an increased risk of ACS would be observed also in individuals with a similar genetic set-up and background as the patients with RA, such as their siblings.

Objectives: To investigate any potential shared susceptibility between RA and ACS by estimating the risk of ACS in full siblings of patients with (vs. without) RA. Methods: We used the Swedish Rheumatology Quality register (SRQ) to identify an early RA cohort diagnosed between 1996-2015, which was linked to the Swedish Multigeneration Register, Patient Register, the Cause of Death register, and the Total Population Register. Through this, we sampled five general population comparator subjects to each patient with RA, matched by birth year and sex, and identified all full siblings to patients with RA and for their comparator subjects born within five years of their index case. The comparators, and all siblings, were required to be alive and living in Sweden at the time of the index patient's RA diagnosis (=start of follow-up). All unique individuals were then followed for ACS (defined as first ever hospitalisation for ACS (ICD10: I21 or I20.0) or MI listed as the cause of death), and censored at death, migration, RA diagnosis (for non-RA subjects) or the end of the study (Dec 31 st 2015). We calculated hazard ratios (HR) using a Cox proportional hazards model, adjusting for age, sex and calendar period of diagnosis. Confidence intervals $(\mathrm{Cl})$ were estimated using a robust sandwich estimator.

Results: We identified 7492 patients with RA who had 10671 full siblings, and 35120 population comparator subjects with 47137 full siblings. The HR for ACS was 1.44 (95\% Cl: 1.25 to 1.66$)$ and $1.23(95 \% \mathrm{Cl}: 1.09$ to 1.40$)$ for patients with $\mathrm{RA}$ and their siblings, respectively, compared to the comparator subjects. A direct comparison between the RA patients and their RA-free siblings confirmed the familial association between RA and ACS, HR 1.19 (95\% Cl: 1.02 to 1.38$)$.

Conclusions: The increased risk of ACS in siblings of patients with RA a) provide evidence of shared susceptibility between RA and ACS, the nature of which needs to be further investigated, and $b$ ) suggests that to bring down the $C V$ risk in RA to that in the general population, cardiopreventive measures must go beyond optimised RA disease control.

\section{REFERENCE:}

[1] Holmqvist M, Ljung L, Askling $\mathrm{J}$ Acute coronary syndrome in new-onset rheumatoid arthritis: a population-based nationwide cohort study of time trends in risks and excess risks, Ann Rheum Dis 2017

Disclosure of Interest: H. Westerlind: None declared, M. Holmqvist: None declared, L. Ljung: None declared, T. Frisell: None declared, J. Askling Grant/ research support from: Johan Askling has or has had research agreements with Abbvie, BMS, MSD, Pfizer, Roche, Astra-Zeneca, Eli Lilly, Samsung Bioepis, and UCB, mainly in the context of safety monitoring of biologics via ARTIS. Karolinska Institutet has received remuneration for JA participating in advisory boards arranged by Pfizer and Eli Lilly.

DOI: 10.1136/annrheumdis-2018-eular.3518

\section{OP0137 ASSESSING THE RISK OF RA PATIENTS FOR COMORBID CONDITIONS THROUGH A STRUCTURED NURSE-LED INTERVIEW - THE ERIKO STUDY}

K. Krueger ${ }^{1}$, R. Eder ${ }^{2}$, C. Mueller ${ }^{3}$, K. Rietzler ${ }^{3} .{ }^{1}$ Rheumatologisches Praxiszentrum St. Bonifatius, München; ${ }^{2}$ Rheumapraxis am Spitaltor, Deggendorf, ${ }^{3} \mathrm{AbbVie}$ Deutschland GmbH and Co. KG, Wiesbaden, Germany

Background: Assessment of comorbidities in rheumatoid arthritis (RA) has proven to be difficult for the rheumatologist due to lack of time leading to considerable deficits. Nurse-led programs on RA comorbidity management might solve this problem and have been reported to be beneficial. ${ }^{1}$

Methods: The cluster randomised multicentre study ERIKO longitudinally assessed the general health status of patients with RA in Germany applying a nurse-led scoring algorithm for individual risk profiles (ERIKO-Score). The ERIKO-Score is a composite score evaluating the following risk factors: cardiovascular (CV) risk (ESC-guideline), infection risk (RABBIT risk calculator), vaccination status (guideline), fracture risk (FRAX), tooth status (PSI), depression (PHQ-9) and health-related quality of life (hrQoL, RAID). The outcome was translated into a three-level ordinal score defined by the categories low, intermediate or high risk with 0,1 and 2 points, respectively. Afterwards the treating rheumatologist discussed the outcomes with the patient. The primary endpoint, the change in ERIKO-Score between baseline and after one year of follow-up as a consequence of the structured assessment (active arm) was assessed using a mixed model in order to account for a random centre effect. Furthermore, comorbidity management in the active arm versus expert guided assessment following standard of care (SOC, control arm) was evaluated using Fisher's exact test and the CochranArmitage trend test.

Results: The analysis included 341 patients from 42 rheumatology centres with 210 patients in the active arm (mean disease duration 12.0 years (sd 8.8), mean DAS28 2.6 (sd 1.1)) and 131 patients in the control arm (mean disease duration 9.7 years (sd 7.4), mean DAS28 2.6 (sd 1.2)). After one year of follow-up the structured assessment led to a significant mean decrease in total ERIKO-Score by $-0.45(95 \% \mathrm{Cl}$ ranging from -0.67 to $-0.23, \mathrm{p}<0.001)$. This decrease was driven by an improvement in vaccination status, tooth status and depression risk. No difference in comorbidity management was detected between the structured assessment and SOC. At the end of follow-up, the mean ERIKO-Scores for both arms were 4.85 with $95 \%$ Cls ranging from 4.59 to 5.11 and 4.47 to 5.24 for the active arm and the control arm, respectively. A significant improvement in risk categorisation after one year of follow-up was observed for vaccination status and depression risk with better outcomes in the active arm as compared to the control arm.

Conclusions: A nurse-led comorbidity risk assessment in rheumatology practices resulted in a significant improvement after one year of follow-up. However improvement was small and there was no benefit compared to expert-opinion based SOC. In clinical routine, applying the ERIKO-Score based on validated tools seems to be feasible. Nurse-led comorbidity assessment seems to be able to reduce existing deficits.

\section{REFERENCE:}

[1] Dougados M, et al. Ann Rheum Dis 2015;74:1725-33.

Acknowledgements: This study was sponsored by AbbVie Deutschland $\mathrm{GmbH}$ and Co KG. AbbVie contributed to the study design, interpretation of data, writing, reviewing, and approving the abstract. The authors wish to thank Dr. Daniela Adolf, of StatConsult $\mathrm{GmbH}$ for conducting the statistical analysis and reviewing the manuscript. AbbVie provided funding to StatConsult GmbH for this work.

Disclosure of Interest: K. Krueger Consultant for: AbbVie Deutschland GmbH and Co. KG., Speakers bureau: AbbVie Deutschland GmbH and Co. KG, R. Eder Consultant for: AbbVie Deutschland GmbH and Co. KG., C. Mueller Shareholder of: AbbVie Deutschland GmbH and Co. KG., Employee of: AbbVie Deutschland $\mathrm{GmbH}$ and Co. KG, K. Rietzler Shareholder of: AbbVie Deutschland GmbH and Co. KG., Employee of: AbbVie Deutschland GmbH and Co. KG. DOI: 10.1136/annrheumdis-2018-eular.1268

\begin{tabular}{|l|l}
\hline OP0138 & INVESTIGATION OF BIOPSYCHOSOCIAL \\
ASSESSMENTS IN PATIENTS WITH RHEUMATIC \\
DISEASE
\end{tabular}

N.B. Karaca ${ }^{1}$, G. Arın ${ }^{1}$, A. Özçadırcl ${ }^{1}$, F.B. Oflaz ${ }^{1}$, S. Apraş Bilgen ${ }^{2}$, E. Ünal ${ }^{1}$ ${ }^{1}$ Department of Physiotherapy and Rehabilitation, Hacettepe University Faculty of Health Sciences; ${ }^{2}$ Department of Internal Medicine-Rheumatology, Hacettepe University Faculty of Medicine, Ankara, Turkey

Background: The daily living activities, functions, depression and anxiety of patients with rheumatic diseases can be affected at various levels. ${ }^{1}$ This condition explains the biopsychosocial dimension of chronic pain, it also requires the multidimensional assessment of the disease. ${ }^{2}$

Objectives: The aim of this study was to investigate biopsychosocial assessments of patients with rheumatic diseases.

Methods: Patients with rheumatic diseases were included in this prospective study. Demographic informations of patients were recorded. Health Assessment Questionnaire (HAQ) was used to assess daily living activities, the Hospital Anxiety and Depression Scale (HADS) was used to assess the anxiety and depression levels and Cognitive Exercise Therapy Approach Scale (the authors request that the abbreviation stay as 'BETY' as the original in Turkish) was used to assess biopsychosocial status of the patients. ${ }^{3}$ Data obtained from 352 patients were analysed by correlation analysis.

Results: 352 patients ( $82.4 \%$ women, $17.6 \%$ men, mean age: $46.01 \pm 11.6$ years) were included in this study. Descriptives of the assessment results were given in table 1. There was a statistically significant correlation between the BETY and the 